\title{
QUOTIENTS OF VALUATED VECTOR SPACES
}

\author{
PAUL HILL
}

\begin{abstract}
This paper has three purposes. The first is to present a direct example of a quotient of an injective space that is not itself injective. The second is to demonstrate that Theorem 3 in [2] is false. An $s$-dense subspace $S$ of a free space $F$ is not free by virtue of $F / S$ having only values that are cofinal with $\omega$. Finally, we furnish a valid proof, independent of the aforementioned Theorem 3, that a subspace $V$ of a free space is free if $V$ satisfies Fuchs' countability condition.
\end{abstract}

1. Quotients of injective valuation spaces. In a ground-breaking paper on valuated vector spaces by L. Fuchs [1], Theorem 7 states that quotients of injective valued vector spaces are likewise injective. Unfortunately, this theorem is erroneous. Its falsity was discovered first by F. Richman and E. A. Walker [5] and subsequently by E. White [6] as well as the present author [3]. The theorem was partially salvaged by Fuchs in [2]. One consequence of Theorem 7 (if it were true) is that every valuated vector space has projective dimension at most one. The fact that Theorem 7 in [1] is false was discovered (by all authors mentioned above) indirectly through the discovery of spaces with projective dimension greater than 1 . We shall give a direct counterexample, by exhibiting a subspace $W$ of a product $P$ of homogeneous spaces such that $P / W$ is not injective.

The value of an element $x$ is denoted herein by $|x|$. Recall that if $W$ is a subspace of $V$ the valuation on the quotient space $V / W$ is given by

$$
|x+W|=\sup \{|x+w|: w \in W\}
$$

for any $x \in V$. For convenience of proof that our example is valid, we shall employ the notion of a separable subspace which was introduced in [3].

Definition 1. A subspace $W$ of $V$ is separable if $|x+W|=\sup \left\{\left|x+w_{n}\right|\right\}$ for some countable sequence $\left\{w_{n}\right\}$ of elements in $W$.

Definition 2. A valuated vector space $W$ is said to be absolutely separable if it is separable in every containing space.

LEMMA 1 [3]. Any free space is absolutely separable.

The next result is of independent interest and is essential for our main result.

LEMma 2. Any separable subspace of an injective valuated vector space is absolutely separable.

Received by the editors February 15, 1979 and, in revised form, December 9, 1979.

1980 Mathematics Subject Classification. Primary 18G05, 18G20; Secondary 20K10.

Key words and phrases. Valuation, valuated vector space, free space, quotient of injective, s-dense subspace, separable subspace, projective dimension greater than one, criterion for freeness. 
Proof. Suppose that $W$ is separable in the injective space $E$ and that $W$ is a subspace of some other space $V$. Assume that $W$ is not separable in $V$. Consequently, we can choose $x \in V$ so that

$$
|x+W|>\sup \{|x+c|: c \in C\}
$$

for every countable subset $C$ of $W$. In other words, we can choose $x \in V$ so that $W$ is not separable in $\langle W, x\rangle$. Let $\varphi: W>\rightarrow E$ denote the inclusion map. Since $E$ is injective, $\varphi$ can be extended (in the category of valuated vector spaces) to a map from $\langle W, x\rangle$ into $E$. Continue to denote the extension by $\varphi$ and let $y=\varphi(x)$. Since $\varphi$ is a map, the inequality of values $|c x+w|<|c y+w|$ holds for all $w \in W$ and all scalars $c$. We shall demonstrate that equality actually holds. There is no loss of generality in assuming that $c=1$ since $|c x|=|x|$ for every nonzero scalar $c$. Since $W$ is not separable in $\langle W, x\rangle$, there exists $w^{\prime} \in W$ such that $\left.\left|x+w^{\prime}\right|\right\rangle$ $|x+w|$. For a fixed $w \in W$ let $|x+w|=\lambda$ and observe that $\left|w^{\prime}-w\right|=\lambda$. Moreover, $\left|y+w^{\prime}\right| \geqslant\left|x+w^{\prime}\right|>\lambda$, which precludes the inequality $|y+w|>\lambda$ since it would lead to $\left|w^{\prime}-w\right|>\lambda$ and a contradiction. Hence $|x+w|=|y+w|$ for all $w \in W$, and $\varphi$ must be an imbedding of $\langle W, x\rangle$ into $E$. However, it is now impossible for $W$ not to be separable in $\langle W, x\rangle$ because $W$ is separable in $E$, and the lemma is proved.

We are now prepared to present directly an example of a quotient of an injective that is not itself injective.

EXAMPLE. For each pair $(\alpha, \beta)$ of countable ordinals with $\alpha<\beta$, let $x_{\alpha, \beta}$ designate a generator having value $\alpha$ and let $F=\Sigma\left\langle x_{\alpha, \beta}\right\rangle$ be the free space produced by the coproduct of the one dimensional spaces $\left\langle x_{\alpha, \beta}\right\rangle$. Equivalently, if $R$ denotes the usual relation $<$ on $\omega_{1}$, then $F$ is simply the free valuated vector space based on the set $R$ where the value assigned to $(\alpha, \beta) \in R$ is its first component.

For every fixed countable ordinal $\alpha$, let

$$
H_{\alpha}=\sum_{\alpha<\beta<\omega_{1}}\left\langle x_{\alpha, \beta}\right\rangle \text { and } E=\prod_{\alpha<\omega_{1}} H_{\alpha} .
$$

The homogeneous space $H_{\alpha}$ is injective and therefore so is the product $E$. It is worthwhile to observe that

$$
F=\sum H_{\alpha} \subseteq \prod H_{\alpha}=E .
$$

All we need to do is find a subspace $W$ of $E$ so that $E / W$ is not injective. A suitable subspace $W$ of $F \subseteq E$ is defined, with $\lambda$ fixed, as follows.

$$
W=\left\{\sum c_{\alpha, \beta} x_{\alpha, \beta} \in F: \sum_{\mu>\lambda} c_{\lambda, \mu}=\sum_{\mu<\lambda} c_{\mu, \lambda}\right\} .
$$

In the above summations, $\lambda$ is arbitrary but fixed; in other words, the condition $\Sigma_{\mu>\lambda} c_{\lambda, \mu}=\Sigma_{\mu<\lambda} c_{\mu, \lambda}$ must hold for each countable ordinal $\lambda$ where $\mu$ is the summation variable. The vacuous sum is, of course, understood to be zero. Thus $\Sigma c_{\alpha, \beta} x_{\alpha, \beta} \in W$ requires, in particular, that $\Sigma_{\mu>0} c_{0, \mu}=0$. A typical element of $W$ is $x_{\alpha, \beta}-x_{\alpha, \gamma}+x_{\beta, \gamma}$ where $\alpha<\beta<\gamma$. In particular, $x_{0, \alpha}-x_{0, \beta}+x_{\alpha, \beta}$ is always in $W$ whenever $0<\alpha<\beta$. The fact that $E / W$ is not injective is the essence of the following. 
TheOREM 1. $E$ is injective, but the quotient $E / W$ is not injective.

Proof. We have already observed that the product $E=\Pi H_{\alpha}$ is injective. In order to prove that $E / W$ is not injective, it suffices by Lemma 2 to show that $F / W$ is a separable subspace of $E / W$ but that $F / W$ is not absolutely separable. The separability of $F / W$ in $E / W$ comes easily as a consequence of Lemma 1 (which implies that $F$ is separable in $E)$ and the isomorphism $(E / W) /(F / W) \cong E / F$. In order to show that $F / W$ is not absolutely separable, it is convenient to identify $F / W$ with a particular known nonseparable subspace of some space. Toward that end, let $B=\Sigma_{\alpha<\omega_{1}}\left\langle b_{\alpha}\right\rangle$ be the free space with $\left|b_{\alpha}\right|=\alpha$ for each countable ordinal $\alpha$. Denote by $A$ the subspace of $B$ spanned by the elements of the form $b_{0}-b_{\alpha}$, $A=\left\langle b_{0}-b_{\alpha}\right\rangle_{\alpha<\omega_{1}} \subseteq B$. Since $\left|b_{0}+A\right|=\omega_{1}, A$ is certainly not separable in $B$. If we can show that $F / W$ is isometric to $A$, the proof of the theorem will be finished.

The correspondence $\pi$ defined by $b_{0}-b_{\alpha} \rightarrow x_{0, \alpha}+W$, or rather more completely by

$$
\sum c_{\alpha}\left(b_{0}-b_{\alpha}\right) \rightarrow \sum c_{\alpha} x_{0, \alpha}+W
$$

is at least a linear transformation (if not a valuation map) from $A$ into $F / W$ since $\left\{b_{0}-b_{\alpha}\right\}_{\alpha<\omega_{1}}$ is linearly independent in $A$. Moreover, since $x_{\alpha, \beta}+W=$ $x_{0, \beta}-x_{0, \alpha}+W, \pi$ is onto. It remains only to show that $\pi$ preserves values since that automatically implies that $\pi$ is one-to-one. To demonstrate that values are preserved, observe that if

$$
0<\alpha(1)<\alpha(2)<\cdots<\alpha(n)<\beta<\omega_{1},
$$

then

$$
\sum_{i=1}^{n} c_{\alpha(i)} x_{0, \alpha(i)}+W=\left(\sum_{i=1}^{n} c_{\alpha(i)}\right) x_{0, \beta}-\sum_{i=1}^{n} c_{\alpha(i)} x_{\alpha(i), \beta}+W .
$$

Furthermore, if $c_{\alpha(i)} \neq 0$ (as we may assume), the value of the element on the right-hand side of the equation is either 0 or $\alpha(1)$ depending on whether $\sum_{i=1}^{n} c_{\alpha(i)}$ is different from zero or not. The point is that the value of the representative on the right-hand side cannot be increased by adding an element of $W$. Hence the value of $\sum_{i=1}^{n} c_{\alpha(i)} x_{0, \alpha(i)}+W$ is precisely the same as the value of $\sum_{i=1}^{n} c_{\alpha(i)}\left(b_{0}-b_{\alpha(i)}\right)$, and the theorem is proved.

2. Dense subspaces of free spaces. In this section we demonstrate that an $s$-dense subspace $S$ of a free space $F$ need not be free solely by virtue of the values of $F / S$ all being cofinal with $\omega=\omega_{0}$, contrary to Theorem 3 in [2].

A subspace $W$ of $V$ is $s$-dense in $V$ if for each $x$ in $V$ not in $W$ there exists $w \in W$ such that $|x-w|>|x|$. Equivalently, $W$ is $s$-dense in $V$ if there is no subspace $S$ of $V$ properly containing $W$ as a direct summand in the valuated sense.

The proofs of the next three propositions are omitted since they are routine. The propositions are stated here only because they provide an outline for the proof of the main result of this section.

Proposition 1. If $A_{i}$ is $s$-dense in $B_{i}$ for each $i$, then the coproduct $\Sigma A_{i}$ is s-dense in the coproduct $\Sigma B_{i}$. 
Proposition 2. Let $\beta$ be a limit ordinal cofinal with $\omega$ and let $\{\alpha(n)\}$ be a strictly increasing sequence of ordinals whose limit is $\beta$. Then $S=\left\langle x_{\alpha(0)}-x_{\alpha(n)}\right\rangle$ is $s$-dense in the free space $F$ determined by the coproduct $F=\Sigma\left\langle x_{\alpha(n)}\right\rangle$ where $\left\langle x_{\alpha(n)}\right\rangle$ is the one-dimensional space having value $\alpha(n)$. Moreover, $F / S$ is a one-dimensional space having the preassigned value $\beta$.

Combining the preceding propositions, we obtain the following.

Proposition 3. If $V$ is a free space whose values (with the possible exception of $\infty$ ) are all cofinal with $\omega$, then $V=F / S$ is the quotient of a free space $F$ by an s-dense subspace $S$.

We shall now consider the set of all ordinals less than $\omega_{2}$ that are cofinal with $\omega$. Let

$$
\Lambda=\left\{\lambda<\omega_{2}: \operatorname{cof}(\lambda)=\omega\right\} .
$$

Set $V=\Sigma_{\lambda \in \Lambda}\left\langle x_{\lambda}\right\rangle$ where $\left|x_{\lambda}\right|=\lambda$, and let $W=\left\langle x_{\omega}-x_{\lambda}\right\rangle_{\lambda \in \Lambda}$. Using an argument almost identical to that of E. White in [6], we can establish

Proposition 4. $W$ is not the quotient of a free space by a free space.

Proof. Even though the proof is essentially the same as that of White [6], for convenience of the reader we shall provide an outline. Assume that $W$ is a QFF space, that is, assume that $W$ is the quotient of a free space by a free space. According to Theorem 5 in [3], it follows that $W$ must have a smooth ascending chain of separable subspaces $\left\{W_{\rho}\right\}$ where $\operatorname{dim}\left(W_{\rho}\right)<\aleph_{1}$ for each $\rho$ and where $W=\cup W_{\rho}$. However, for some $\rho$ it must be the case that

$$
W_{\rho}=\left\langle x_{\omega}-x_{\lambda}\right\rangle_{\lambda \in \Lambda: \lambda<\mu}
$$

for some $\mu<\omega_{2}$ having cofinality $\omega_{1}$; the fact that $\mu$ is not contained in $\Lambda$ is beside the point. This description of $W_{\rho}$ yields a contradiction since the space on the right-hand side of the equation is obviously not separable in $W$. We conclude that $W$ cannot be a QFF space; in the terminology of [4], $W$ has projective dimension $\geqslant 2$.

THEOREM 2. There exists a free space $F$ and an $s$-dense subspace $S$ such that all the values of $F / S$ are cofinal with $\omega$, yet $S$ is not free.

Proof. Let $W=\left\langle x_{\omega}-x_{\lambda}\right\rangle_{\lambda \in \Lambda}$ be as in Proposition 4. Hence, we know that $W$ is not a QFF space and we know that all the values, with the exception of $\infty$, of elements of $W$ are cofinal with $\omega$. Consider the canonical exact sequence

$$
K>F_{W} \rightarrow W,
$$

where $F_{W}$ is the free space based on $W$. Since $F_{W}$ is based on $W$, the value of each nonzero element of $F_{W}$ is cofinal with $\omega$. By Proposition 3, there exist a free space $F$ and an $s$-dense subspace $S$ such that $F / S=F_{W}$. Choose the subspace $T$ of $F$ containing $S$ so that $T / S=K \subseteq F_{W}$. Trivially, $T$ is $s$-dense in $F$ since $S$ is and $T \supseteq S$. But $T$ cannot be free because $F / T=W$ is not a QFF space. 
REMARK. In the preceding theorem the cofinality of $\infty$ is really of no consequence. However, we can make $\operatorname{cof}(\infty)=\omega$ also if we set $\left|y_{n}\right|=\omega_{2}+n$ and take the $s$-dense subspace $T+\Sigma\left\langle y_{n}\right\rangle$ of the free space $F+\Sigma\left\langle y_{n}\right\rangle$.

3. A certain criterion for freeness. A valuated vector space $V$ satisfies Fuchs' countability condition if every ascending chain of values of elements in $V$ is countable. The proof in [2] of the following theorem was based on the erroneous result discussed in \$2; however, the theorem itself remains valid.

TheOREM 3 [2]. If $H$ is a subspace of a free space $F$ and $H$ satisfies the countability condition, then $H$ is free.

Proof. The proof is by induction on $\operatorname{dim}(F)$. If $\operatorname{dim}(F)<\aleph_{0}$, then $\operatorname{dim}(H)<$ $\aleph_{0}$, and therefore $H$ is free. Assume $\operatorname{dim}(F) \geqslant \aleph_{1}$ and set $m=\operatorname{dim}(F)$. Since $H$ satisfies the countability condition, it follows at once that $H$ is separable. Therefore, by Lemma 1 in [3], there exists a smooth ascending chain of summands $F_{\alpha}$ of the free space $F$ such that the following conditions are satisfied for each $\alpha$.

(0) $F_{0}=0$

(1) $\operatorname{dim}\left(F_{\alpha}\right)<m$.

(2) $F_{\alpha} \| H$.

Recall that $A \| B$ means that for each pair $(a, b) \in A \times B$ there exists $c$ in $A \cap B$ such that $|a+c| \geqslant|a+b|$. Set $H_{\alpha}=H \cap F_{\alpha}$ and observe, in view of condition (2), the inclusion of $H_{\alpha+1} / H_{\alpha}$ in a free space by virtue of the natural isomorphism

(3) $H_{\alpha+1} / H_{\alpha}=H_{\alpha+1} / F_{\alpha} \cap H_{\alpha+1}=\left\langle F_{\alpha}, H_{\alpha+1}\right\rangle / F_{\alpha} \subseteq F_{\alpha+1} / F_{\alpha}$.

Since $H$ satisfies the countability condition so does $H_{\alpha+1} / H_{\alpha}$. Condition (1) implies that $\operatorname{dim}\left(F_{\alpha+1} / F_{\alpha}\right)<m$. Hence by the induction hypothesis, $H_{\alpha+1} / H_{\alpha}$ is free. Since $\left\{F_{\alpha}\right\}$ is a smooth ascending chain, $\left\{H_{\alpha}\right\}$ is a smooth ascending chain too. Moreover, since $F_{\alpha}$ is nice in $F$ and since $H \| F_{\alpha}$, it follows that $H_{\alpha}$ is nice in $H$. Therefore, $H_{\alpha}$ is a summand of $H_{\alpha+1}$, so $H_{\alpha+1}=H_{\alpha}+C_{\alpha}$ where $C_{\alpha} \cong H_{\alpha+1} / H_{\alpha}$ is free. Since $H=\Sigma C_{\alpha}$, the theorem is proved.

\section{REFERENCES}

1. L Fuchs, Vector spaces with valuations, J. Algebra 35 (1975), 23-38.

2. Subfree valued vector spaces, Lecture Notes in Math., Vol. 616, Springer-Verlag, Berlin and New York, 1977, pp. 158-167.

3. P. Hill, Criteria for freeness in groups and valuated vector spaces, Lecture Notes in Math., Vol. 616, Springer-Verlag, Berlin and New York, 1977, pp. 140-157.

4. P. Hill and $\mathrm{E}$. White, The projective dimension of valuated vector spaces (preprint).

5. F. Richman and E. A. Walker, Valuated groups, J. Algebra 56 (1979), 145-167.

6. E. White, On the homological dimension of valuated vector spaces, Canad. Bull. Math. (to appear).

Department of Mathematics, Auburn University, Auburn, Alabama 36830 